\title{
Mobile learning multidispositivo en la enseñanza universitaria a distancia
}

\section{Extracto:}

La evolución de las tecnologías de la información y la comunicación (TIC) en los últimos años ha tenido un gran efecto en la manera de enfocar la enseñanza, sobre todo en la modalidad de mobile learning. Hoy en día, la mayoría de los estudiantes cuentan con multitud de dispositivos para conectarse a internet y quieren utilizarlos todos para un aprendizaje en cualquier momento y en cualquier lugar. Para afrontar este reto, la Universidad a Distancia de Madrid (UDIMA) se propuso implementar aplicaciones (apps) complementarias al estudio para este entorno multidispositivo. Se comenzó con una prueba piloto realizada para sistema operativo Android y válida tanto para smartphones como para tablets. A partir de ella, el siguiente paso fue el desarrollo de una aplicación web (web app) con diseño web adaptable, que es compatible con la práctica totalidad de los nuevos dispositivos móviles que existen en el mercado. Actualmente, se ha extendido esta iniciativa y ya se cuenta con cinco aplicaciones web para diversas asignaturas tanto de grado como de máster. Los estudiantes creen que son de gran ayuda para el aprendizaje y que aportan valor añadido a la enseñanza. Los módulos de casos prácticos y contenido multimedia son los más valorados. Los estudiantes querrían también un sistema de avisos de la asignatura.

Palabras claves: mobile learning, multidispositivo, web apps, diseño web adaptable, enseñanza universitaria a distancia.

\footnotetext{
F. D. de la Peña Esteban, profesor ayudante doctor de la Escuela de Ciencias Técnicas e Ingeniería de la Universidad a Distancia de Madrid (UDIMA).

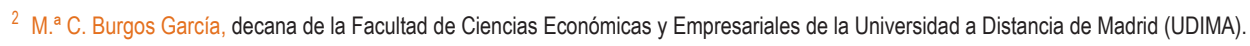

${ }^{3}$ M. ${ }^{a}$ A. Simón Rodríguez, profesora asociada doctora de la Escuela Politécnica de la Universidad Alfonso X el Sabio.
} 


\section{Multidevice}

\section{moble}

learning in

\section{open}

\section{university teaching}

\section{Abstract:}

The development of information and communication technologies (ICT) throughout the past years has had a great effect on the way learning is approached, specially on mobile learning. Nowadays, most students rely on numerous devices with internet connection, which they hope to be able to use anywhere and anytime as learning tools. Facing this challenge, Madrid Open University (UDIMA) set out to implement applications (apps) complementary to conventional studying techniques in this multidevice environment. Firstly, a pilot trial was carried out for Android operating system, available for both smartphones and tablets. Building on this study, the next step was the development of a web application (web app) with a responsive web design (RWD), which is practically compatible with all new modern mobile devices. This initiative is currently widespread, and five web apps are available for various assignments for both degree and masters programs. Students find them to be of great help for learning, and consider them to add value to the learning experience. The practical cases modules and multimedia content stand out as the most appreciated. Students would also like to have a notification system for the classes.

Keywords: mobile learning, multidevice, web apps, responsive web design (RWD), distance university education.

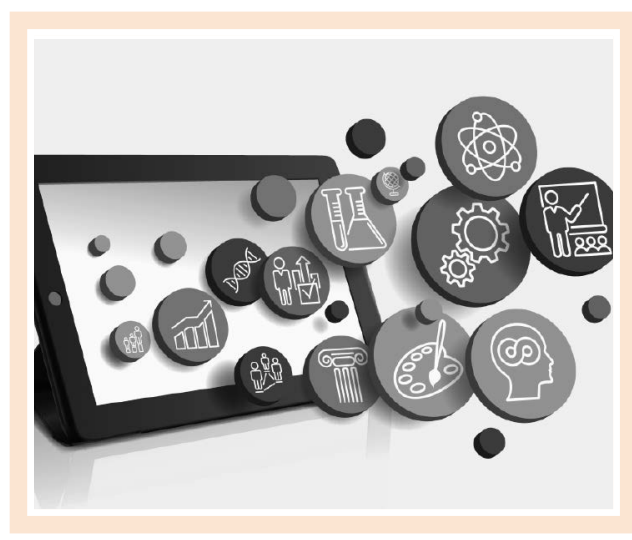

\section{INTRODUCCIÓN}

La educación debe evolucionar con las nuevas TIC. La masificación de los dispositivos móviles (smartphones y tablets, principalmente), en conjunción con las altas velocidades de transmisión de datos, tanto de la conectividad móvil (redes $3 \mathrm{G}$ y $4 \mathrm{G}$ ), como de la banda ancha, proporciona la posibilidad única de poder acceder en un tiempo muy pequeño a gran cantidad de datos. Los estudiantes, y sus profesores, se han dado cuenta de las grandes ventajas que pueden tener si logran utilizar su amplia gama de dispositivos de acceso a internet para el aprendizaje.

En España, según muestra el estudio «Digital in 2016», de la compañía We Are Social, el tráfico web, cuya procedencia son los teléfonos inteligentes, está subiendo año tras año, situándose en la actualidad por encima del $32 \%$. Además, España cuenta con el mayor ratio de usuarios de telefonía móvil, ya que supera el $87 \%$. La conexión móvil a través de redes $3 \mathrm{G}$ y $4 \mathrm{G}$ supera el $74 \%$ de las conexiones móviles que existen en nuestro país.

Los estudiantes, y sus profesores, se han dado cuenta de las grandes ventajas que pueden tener si logran utilizar su amplia gama de dispositivos de acceso a internet para el aprendizaje 
El smartphone se ha convertido en el medio más utilizado en España para acceder a internet, con un porcentaje superior al $85 \%$, según el «Estudio Mobile 2015-Spain», realizado por IAB. Le siguen los ordenadores, tanto portátiles como PC, con un $67 \%$; las tablets, con un $45 \%$; y destaca la irrupción de las smarts TV o televisores inteligentes con un $35 \%$. Este mismo estudio menciona otra nueva realidad, que es el uso que se hace de los smartphones y de las tablets como segunda pantalla, utilizándose simultáneamente con los televisores. También incide en la coexistencia de dos sistemas operativos distintos, destacando Android, con más del $80 \%$ de cuota de mercado, e iOS, de Appple, más minoritario, pero con usuarios muy fieles.

Todos estos datos reflejan una nueva realidad que está cambiando radicalmente el mobile learning (conocido también como m-learning). Existen múltiples definiciones de este concepto, como la de Brazuelo y Cacheiro (2010), quienes lo definen como la aplicación de los dispositivos electrónicos móviles para fines educativos; o la de Molnár (2012), quien habla del mobile learning como el acceso a través de dispositivos móviles a los contenidos de un curso $y$ todos los materiales de enseñanza respectivos. El mobile learning se vislumbra como la siguiente generación de la educación a distancia: permite a los estudiantes un aprendizaje just in time, es decir, que se puede acceder al conocimiento en cualquier momento y en cualquier lugar (Sarrab, Elgamel y Aldabbas, 2012), y puede ser utilizado para el aprendizaje colaborativo a distancia (Ozuorcun y Tabak, 2012). Algunos de los retos a los que se debe hacer frente son cómo cuantificar el aprendizaje conseguido únicamente mediante el mobile learning (Ozuorcun y Tabak, 2012), así como la «resistencia tecnológica» a su uso, tanto por parte de docentes como de estudiantes. También hay que hacer frente a las muy frecuentes actualizaciones de los contenidos utilizados (Gikas y Grant, 2013).

\section{Este entorno multidispositivo, en el que coexisten diversos sistemas operativos, y en el que además los tamaños de las pantallas son tan diversos, requiere una solución unificada}

Con todo lo expuesto, la universidad a distancia actual debe hacer frente a estos retos mediante el uso de aplicaciones (apps) para todos los tipos distintos de dispositivos móviles al mismo tiempo. Este entorno multidispositivo, en el que coexisten diversos sistemas operativos, y en el que además los tamaños de las pantallas son tan diversos, requiere una solución unificada.

El objetivo del presente trabajo es mostrar cómo se ha implantado el mobile learning en cinco asignaturas de la UDIMA mediante el uso de aplicaciones web (web apps) con diseño web adaptable, proporcionando un mobile learning multidispositivo en la enseñanza universitaria a distancia. Estas aplicaciones están concebidas como complementarias al Aula Moodle de cada una de las asignaturas.

La estructura que hemos seguido en este trabajo es la siguiente. En el epígrafe 2 vamos a realizar un análisis de la literatura existente en el uso para dispositivos móviles de aplicaciones educativas especialmente creadas para una determinada materia. En el epígrafe 3 detallaremos la metodología propuesta. En el epígrafe 4, como apoyo al estudio, mostraremos los resultados alcanzados en la percepción que tienen los estudiantes de este tipo de aplicaciones. Para finalizar, en el epígrafe 5 expondremos las conclusiones obtenidas.

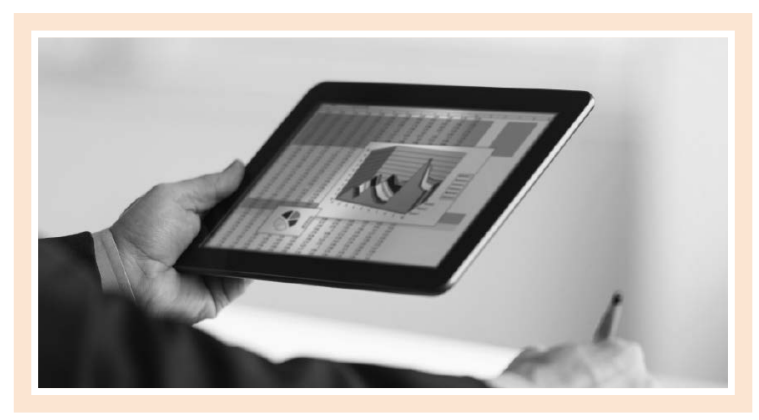




\section{MOBILE LEARNING EN LA ENSEÑANZA UNIVERSITARIA: APLICACIONES}

En este epígrafe vamos a realizar una revisión bibliográfica de los artículos de investigación que se centran en el uso de aplicaciones (apps) para la implementación de mobile learning en la enseñanza universitaria. En la tabla 1 se hace un resumen de esa bibliografía, con una breve descripción de cada una. Sí es interesante mencionar que existen aplicaciones específicas para la impartición de clases presenciales (classroom apps) y otras exclusivas para el aprendizaje a distancia (distance learning apps).

\begin{tabular}{|c|c|}
\hline & Tabla 1. Resumen de las experiencias previas \\
\hline Autores & Descripción \\
\hline Yang y Wang (2011) & $\begin{array}{l}\text { Propuesta de un servicio de aprendizaje con dispositivos móviles Android para los alumnos y con un sistema ba- } \\
\text { sado en web para los profesores. }\end{array}$ \\
\hline Bidin y Zidin (2012) & Análisis de la importancia de las aplicaciones para el m-learning en el sector educativo. \\
\hline Giemza et al. (2012) & $\begin{array}{l}\text { Propuesta de una arquitectura de soporte heterogéneo en entornos de aprendizaje multidispositivo, pero aplicada } \\
\text { únicamente para las clases presenciales. }\end{array}$ \\
\hline Kousar y Scholar (2013) & Estudio sobre el aprendizaje con teléfonos móviles en la enseñanza a distancia. \\
\hline Isabwe, Reichert y Carlsen (2013) & $\begin{array}{l}\text { En un entorno universitario, estudiaron la realización de valoraciones de trabajos de matemáticas entre los propios } \\
\text { estudiantes con ayuda de tablets. }\end{array}$ \\
\hline Kong (2013) & Diseño de un sistema de aprendizaje móvil [mobile learning system (MLS)] en una plataforma Android. \\
\hline Oberer y Erkollar (2013) & Uso de tablets para una asignatura de Marketing mediante la utilización de redes sociales y Hangout. \\
\hline Gordillo et al. (2013) & Propuesta de creación de una herramienta de autor para diseñar contenidos interactivos para múltiples dispositivos. \\
\hline Martin y Ertzberger (2013) & Impartición de una lección de arte para iPad/iPod y su interacción en un museo. \\
\hline Mamat y Azmat (2013) & $\begin{array}{l}\text { Desarrollo de una aplicación en sistema operativo Android para aprender cómo se configuran los routers y los swit- } \\
\text { ches a través de dispositivos móviles. }\end{array}$ \\
\hline Setiabudi y Tjahyana (2013) & Propuesta de un modelo teórico de aplicación híbrida para dispositivos móviles, válida para Android y BlackBerry. \\
\hline Hargis et al. (2014) & Análisis DAFO (debilidades-amenazas-fortalezas-oportunidades) a partir de una experiencia en m-learning con iPad. \\
\hline Amery Ibrahim (2014) & Uso del iPad como apoyo pedagógico en la clase en un curso universitario de programación en $C$. \\
\hline De la Peña y Burgos (2015) & $\begin{array}{l}\text { Propuesta de un modelo práctico de aplicación basándose en las preferencias de los alumnos y en la experien- } \\
\text { cia piloto realizada. }\end{array}$ \\
\hline Al-Harrasi et al. (2015) & $\begin{array}{l}\text { Propuesta de un nuevo modelo de tres fases para la creación de aplicaciones adaptadas a mobile learning: fase de } \\
\text { dimensionamiento inicial, fase de desarrollo y fase de creación de contenidos. }\end{array}$ \\
\hline Ekanayake y Samarakoon (2016) & $\begin{array}{l}\text { Experiencia con smartphones, pero en un entorno cerrado de una red privada, utilizando una aplicación web, para } \\
\text { la realización de trabajos en grupo en el aula. }\end{array}$ \\
\hline
\end{tabular}

Fuente: De la Peña, Burgos y Simón (2015) y elaboración propia. 


\section{DISEÑO DE LA EXPERIENCIA Y METODOLOGÍA}

La metodología que se ha seguido es la que explicamos a continuación. Partiendo de una experiencia previa en la que se desarrolló una aplicación para dispositivos con sistema operativo Android, se vio la necesidad de buscar una alternativa válida para todo tipo de dispositivos. Como alternativa, se encontró el diseño de web apps con diseño web adaptable. Posteriormente, se realizó una prueba piloto con web apps, y luego se expandió a cuatro asignaturas más. Por último, se midió la percepción que tenían los estudiantes de este tipo de apps complementarias al estudio.

\subsection{Experiencia previa}

El primer intento de mobile learning con el uso de aplicaciones tuvo lugar en el curso 20132014, cuando se desarrolló una primera aplicación para sistema operativo Android (De la Peña y Prieto, 2014). Fue muy bien valorada por los estudiantes, pero contaba con el inconveniente de que no era compatible con dispositivos con iOS de Apple. A partir de ella se extrajo la idea de que el uso de aplicaciones para dispositivos móviles era el camino correcto, pero había que encontrar una solución multidispositivo.

\section{(...) las aplicaciones web} con diseño web adaptable (...) son aquellas que adaptan los contenidos al tamaño y orientación de pantalla disponible, teniendo también un menú de navegación similar al de una app nativa

\subsection{Elección del tipo de aplicación para un entorno multidispositivo}

De cara a encontrar una solución, se plantearon los siguientes requerimientos a cumplir por las aplicaciones (apps) complementarias al estudio:

- Multidispositivo. Que se pueda utilizar en todos los dispositivos móviles más utilizados (smartphones, tablets).

- Independiente del sistema operativo. Que valga tanto para dispositivos con sistema operativo Android, como iOS, de Apple.

- Adaptable al tamaño de la pantalla. La aplicación debe ser capaz de conocer el tamaño y posición de la pantalla con la que se está visualizando y adaptarse a la misma.

- Un único desarrollo. Que el mismo desarrollo sea válido para los distintos tipos de dispositivos y sistemas operativos.

- Fácil mantenimiento. Que se puedan realizar actualizaciones de la misma de manera sencilla y que no implique descargas por parte del usuario.

- Compatibilidad. Que sea compatible con los dispositivos móviles existentes más utilizados.

El desarrollo y la puesta a disposición del público de aplicaciones nativas para los distintos tipos de dispositivos móviles posibles no cumplen con estos requisitos. Como alternativa se postularon las aplicaciones web con diseño web adaptable, que son aquellas que adaptan los contenidos al tamaño y orientación de pantalla disponible, teniendo también un menú de navegación similar al de una app nativa. Utilizan como lenguaje de programación HTML5, hojas de estilos CSS3 y «media queries» (Baturay y Birtane, 2013). A diferencia de las apps nativas, no es necesaria su publicación en la tienda de aplicaciones correspondiente al sistema operativo del dispositivo. La actualización de la misma se realiza en la nube, sin necesidad de ninguna acción por parte del usuario. Los navegadores que vienen por defecto en prácticamente todos los dispositivos móviles actuales son compatibles con ellas, lo que proporciona un medio idóneo para todo tipo de dispositivos, independientemente de su sistema operativo y del tamaño de pantalla. En función de todas estas características, se optó por las aplicaciones web con diseño web adaptable. 


\subsection{Desarrollo de web apps para la enseñanza universitaria a distancia}

Una vez decidido el tipo de aplicación, se realizó una prueba piloto con la asignatura Dirección de la Producción del grado en Administración y Dirección de Empresas de la UDIMA.

Los módulos genéricos utilizados son los siguientes (véanse figuras 1 a 4):

- Calendario del aula. Refleja las fechas de entrega de las actividades, así como las de realización de sesiones virtuales.

- Vídeos. Engloban todos los vídeos del profesor realizados expresamente para esa asignatura. Están alojados en su canal de YouTube.

- Sesiones virtuales. Son las grabaciones de las distintas sesiones virtuales realizadas con los estudiantes.

- Unidades. Es un resumen adaptado a dispositivos móviles de las distintas unidades de la asignatura.

- Casos prácticos. Se expone la resolución de los distintos casos prácticos de esa área de conocimiento.

- Podcast. Audios en formato MP3 realizados por el profesor como complemento al estudio.

- Test. Son preguntas de repaso para autocomprobación (verdadero/falso).

- Aula. Acceso directo al Aula Moodle de la asignatura.

La elección de estos módulos es debida al estudio previo realizado (De la Peña y Burgos, 2015) y a la tecnología disponible. Debido a la muy buena aceptación que tuvo entre el alumnado, en estos momentos están disponibles para cinco asignaturas, habiendo sido adaptados los módulos a cada una de ellas.
Figura 1. Menú web app

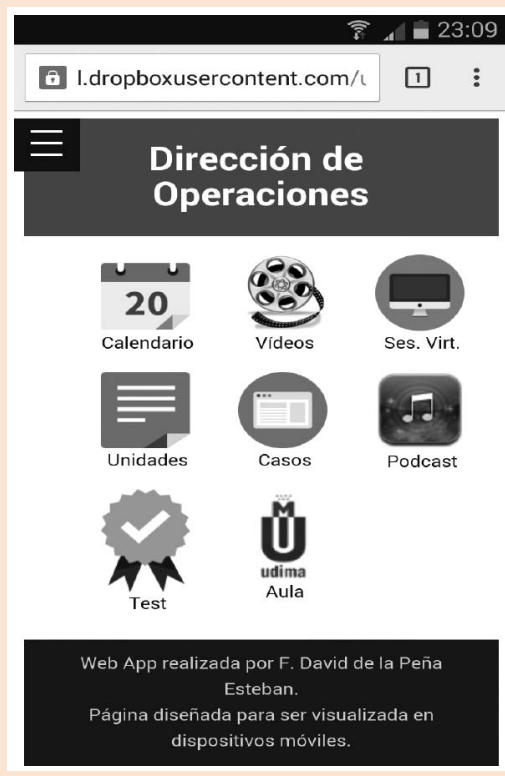

Figura 2. Caso práctico

口

I.dropboxusercontent.com/九 1 :

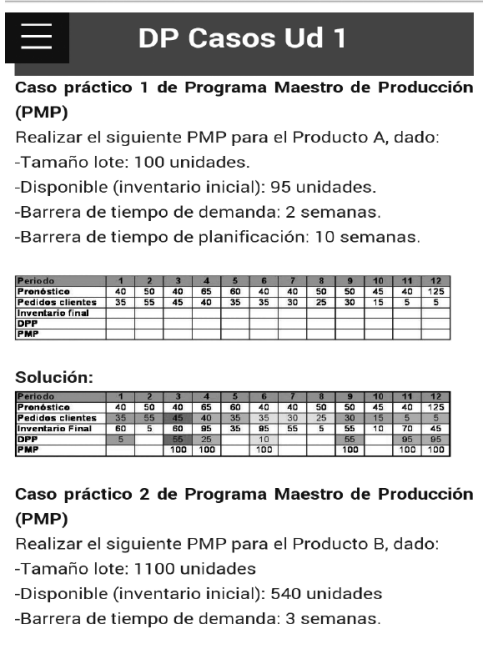

Fuente: elaboración propia. 
Figura 3. Módulo de vídeos

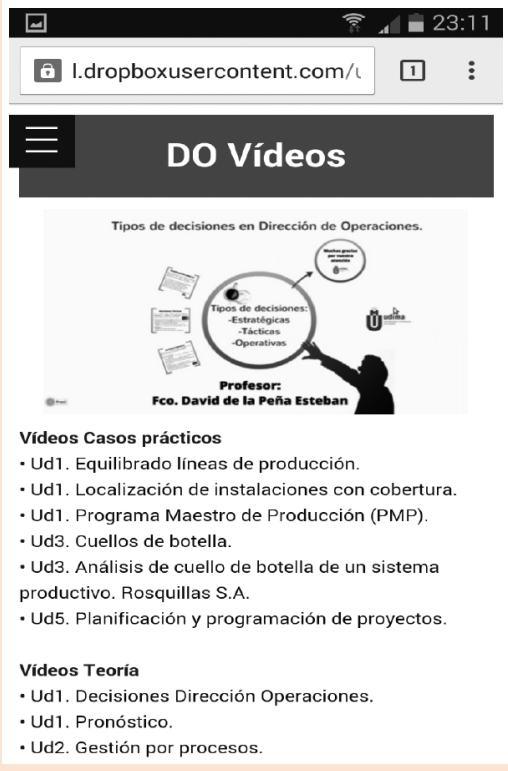

Figura 4. Módulo podcast
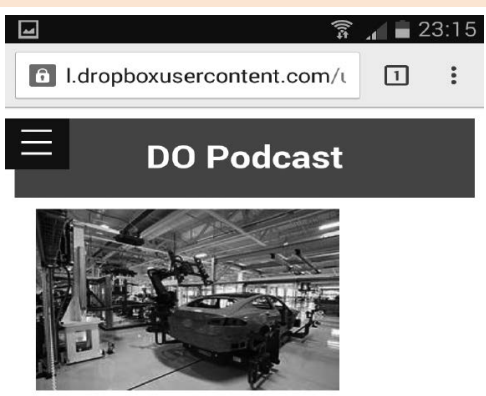

Audios en formato $\mathrm{mp3}$ de la asignatura:

- Unidad 01 - Punto 1 - Decisiones Dirección Operaciones.

\begin{tabular}{lr}
\hline & $2: 11$ \\
\hline & $2: 25$ \\
\hline & $2: 43$ \\
\hline & \\
\hline &
\end{tabular}

Fuente: elaboración propia.

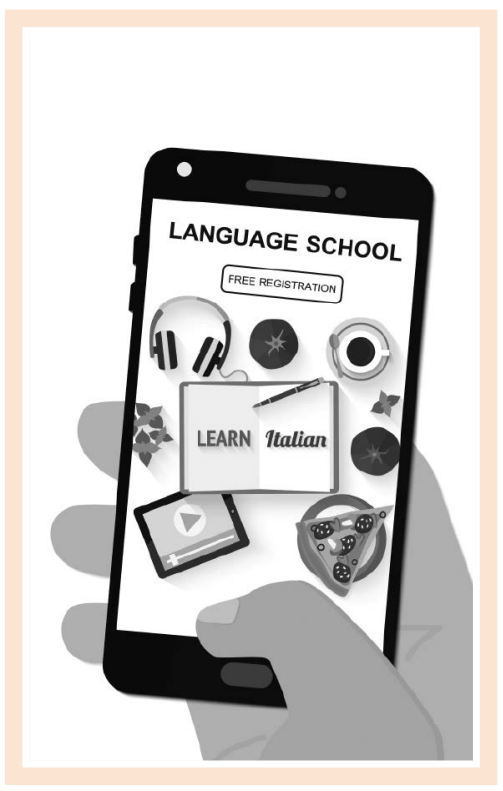

\subsection{Análisis de la percepción del alumnado de las web apps}

Se propuso la realización de la encuesta (véase anexo) a 123 estudiantes procedentes de grado y máster que habían tenido acceso a una web app este semestre. De ellos, contestaron 36. El objetivo de la encuesta era medir la percepción de los estudiantes sobre este tipo de web apps complementarias al estudio. Los resultados están estructurados en seis partes: percepción general sobre las web apps, dispositivos móviles, uso, módulos, así como ventajas y otros aspectos.

Los resultados de la encuesta se pueden ver en el epígrafe 4. En todos aquellos ítems que se valoraban mediante una escala numérica, se utilizó la escala de Likert. Esta escala utiliza cinco niveles de respuesta: de 1 (totalmente en desacuerdo) a 5 (totalmente de acuerdo). 


\section{RESULTADOS ALCANZADOS}

\subsection{Percepción general de las web apps}

La percepción general que tienen los estudiantes hacia el uso de aplicaciones web como complemento a las asignaturas se observa en la tabla 2.

De estos datos, donde todas las medias son superiores a 4, se extrae que, en general, los estudiantes utilizarian las web apps; creen que les ayudarían en el aprendizaje y que aportan valor añadido. También, a partir de las modas y las desviaciones típicas, se puede concluir que existe un pequeño porcentaje de alumnos que es reacio a este tipo de iniciativas.

En relación al tipo de asignatura en la que los alumnos piensan que estas aplicaciones serían más adecuadas, a partir de los datos de la tabla 3, se observa que las consideran válidas tanto para asignaturas teóricas como prácticas.

\subsection{Dispositivos}

Se les preguntó a los alumnos sobre cuáles eran sus dispositivos de acceso preferido. Se les permitía elegir varios a la vez. Según se puede observar en la tabla 4 y en la figura 5 , la gran mayoría prefería acceder con smartphones $(91,7 \%)$, seguido muy de cerca por las tablets $(83,3 \%)$. Hay que reseñar la irrupción de las smart TV (19,4\%) como nuevo dispositivo para el mobile learning.

Como dispositivo que consideran más adecuado para este tipo de web apps, según se detalla en la tabla 5 y en la figura 6 , las tablets obtienen un $77,8 \%$ de los votos.

\section{Tabla 2. Percepción general}

\begin{tabular}{|c|c|c|c|}
\hline Aspectos que se deben valorar & Media & Moda & Desviación \\
\hline $\begin{array}{l}\text { Creo que la existencia de aplicaciones web (web apps) en las } \\
\text { asignaturas mejoraría mi aprendizaje }\end{array}$ & 4,08 & 5 & 1,05 \\
\hline $\begin{array}{l}\text { Estaria dispuesto a utilizar aplicaciones móviles web (web apps) } \\
\text { para mis asignaturas }\end{array}$ & 4,25 & 5 & 1,05 \\
\hline $\begin{array}{l}\text { La existencia de este tipo de aplicaciones móviles aporta un gran } \\
\text { valor añadido a la asignatura }\end{array}$ & 4,17 & 5 & 0,97 \\
\hline
\end{tabular}

Tabla 3. Tipo de asignatura

\begin{tabular}{|c|c|c|}
\hline Tipo de asignatura & Número & Porcentaje \\
\hline Indiferente & 22 & $61,1 \%$ \\
\hline Teóricas & 7 & $19,4 \%$ \\
\hline 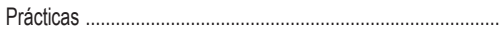 & 6 & $16,7 \%$ \\
\hline Ninguna & 1 & $2,8 \%$ \\
\hline
\end{tabular}

Tabla 4. Tipos de dispositivos preferidos

\begin{tabular}{|c|c|c|}
\hline Tipos de dispositivos de acceso preferido & Número & Porcentaje \\
\hline Smartphone …… & 33 & $91,7 \%$ \\
\hline Tablet & 30 & $83,3 \%$ \\
\hline Smart TV & 7 & $19,4 \%$ \\
\hline Smart watch & 1 & $2,8 \%$ \\
\hline
\end{tabular}

Tabla 5. Dispositivo más adecuado

\begin{tabular}{|c|c|c|}
\hline Dispositivo más adecuado & Número & Porcentaje \\
\hline Tablet ...................... & 28 & $77,8 \%$ \\
\hline 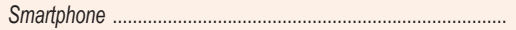 & 8 & $22,2 \%$ \\
\hline
\end{tabular}

Tabla 6. Lugar de utilización

\begin{tabular}{|c|c|c|}
\hline Lugar de utilización de la web app & Número & Porcentaje \\
\hline Estando de viaje & 29 & $80,6 \%$ \\
\hline Medios de transporte ........... & 29 & $80,6 \%$ \\
\hline En esperas (médico, transportes, etc.) ............ & 27 & $75 \%$ \\
\hline Casa ........... & 21 & $58,3 \%$ \\
\hline 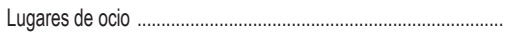 & 10 & $27,8 \%$ \\
\hline Trabajo & 6 & $16,7 \%$ \\
\hline
\end{tabular}

Fuente: elaboración propia 


\subsection{Uso}

Se les cuestionó a los alumnos sobre los lugares en los que ellos consideraban que usarían la aplicación web, siendo los resultados los de la tabla 6 y la figura 7 .

Los estudiantes opinan que la web app se puede usar en numerosas ocasiones, especialmente cuando se está de viaje, en medios de transporte y cuando toca esperar (sea cual sea el tipo de espera). Tampoco es desdeñable que más de la mitad de los encuestados la utilizarían también en casa.

\subsection{Módulos}

Se les pidió a los alumnos que valorasen los posibles módulos que podría contener una web app para una asignatura. Los resultados fueron los de la tabla 7.

\begin{tabular}{|c|c|c|c|}
\hline Caracteristicas & Media & Moda & Desviación \\
\hline Avisos de la asignatura & 4,69 & 5 & 0,79 \\
\hline Casos prácticos autocorregibles & 4,53 & 5 & 0,74 \\
\hline Casos prácticos resueltos & 4,36 & 5 & 0,83 \\
\hline Grabaciones de las sesiones virtuales & 4,28 & 5 & 1,03 \\
\hline Calendario & 4,28 & 5 & 1,16 \\
\hline Videos de casos prácticos & 4,22 & 5 & 1,07 \\
\hline E-mail directo al profesor & 4,22 & 5 & 1,04 \\
\hline Actividades interactivas & 4,17 & 5 & 0,91 \\
\hline Vídeos teóricos & 4,05 & 5 & 1,09 \\
\hline Audios & 4 & 5 & 1,21 \\
\hline Test de autocomprobación & 3,97 & 5 & 1,26 \\
\hline Unidades & 3,89 & 4 & 1,11 \\
\hline Botón de llamada directa a la extensión del profesor & 3,78 & 5 & 1,27 \\
\hline Horario de tutorías & 3,72 & 5 & 1,28 \\
\hline Guía docente & 3,69 & 5 & 1,31 \\
\hline Chat & 3,14 & 3 & 1,40 \\
\hline Integrada con redes sociales (Twitter, Linkedin, etc.) & 2,83 & 1 & 1,42 \\
\hline
\end{tabular}

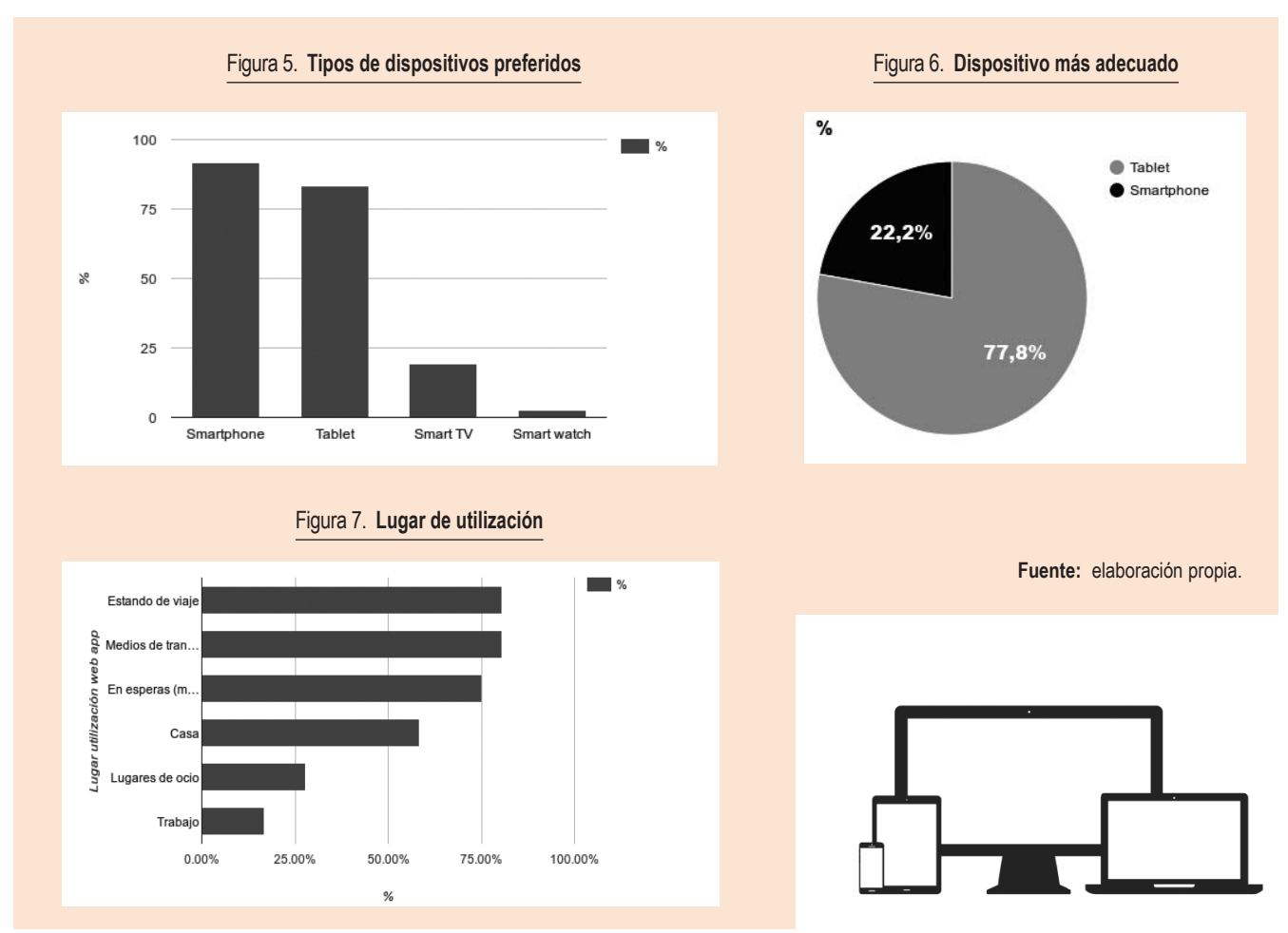


Como principales características que deberían incluir, todas ellas con una nota mayor de 4,25, serían los avisos de la asignatura, casos prácticos autocorregibles y resueltos, así como las grabaciones de las sesiones virtuales y el calendario de la asignatura.

Por el contrario, hay que destacar que los módulos menos deseados son la integración de la aplicación con redes sociales y el chat, con notas inferiores al 3,2.

\section{5. ¿Qué otros aspectos inclui- rías en la aplicación?}

Como características adicionales, los alumnos únicamente destacaron la posibilidad de poder acceder a las calificaciones de las actividades, así como a la secretaría virtual.

\subsection{Ventajas}

Con respecto a las ventajas que este tipo de aplicaciones aportan a los alumnos, una gran parte de ellos comentó el hecho de poder estar conectado en cualquier lugar (movilidad) y en cualquier momento. También destacaron su rapidez, facilidad de acceso y una mejor visualización de contenidos con respecto al Aula Moodle de la asignatura. Otro aspecto que hay que resaltar es que este tipo de aplicaciones les ayudaba a planificar mejor el estudio y la realización de actividades.

\section{Los estudiantes opinan que la web app se puede usar en numerosas ocasiones, especialmente cuando se está de viaje, en medios de transporte y cuando toca esperar (sea cual sea el tipo de espera)}

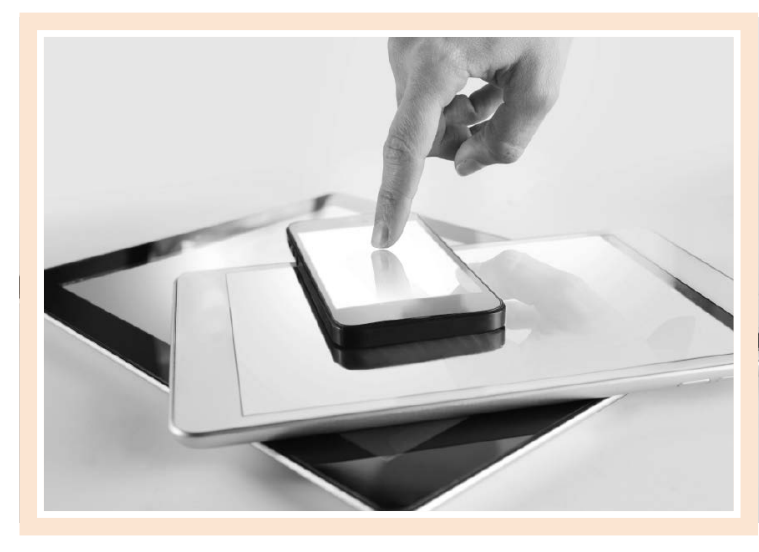

\section{CONCLUSIONES-DISCUSIÓN DE LOS RESULTADOS}

El presente artículo trata sobre la implantación de mobile learning mediante la creación de aplicaciones web (web apps) como complemento a las asignaturas en la enseñanza universitaria a distancia. Este tipo de aplicaciones están realizadas con diseño web adaptable, lo que las hace idóneas para un entorno multidispositivo, en el cual los estudiantes quieren acceder indistintamente con smartphones, tablets, smart TV, etc. Aportan grandes ventajas, al adaptarse a la orientación y al tamaño de la pantalla, al no requerir instalación, al ser compatibles con los nuevos dispositivos móviles y al realizarse un único desarrollo válido para todos los dispositivos.

Se ha realizado una experiencia en la UDIMA, que ha tenido gran acogida entre los estudiantes, y ya está disponible en cinco asignaturas de grado y máster. La percepción que tienen los alumnos de esta clase de aplicaciones es muy positiva, ya que la gran mayoría de ellos afirman que utilizarían este tipo de aplicaciones. También creen que les ayudan en el aprendizaje y que aportan valor añadido a la asignatura.

Piensan que son válidas para todo tipo de asignaturas, tanto teóricas como prácticas, y que se pueden usar en numerosas ocasiones, especialmente cuando se está de viaje, en medios de transporte y en cualquier tipo de espera. Desean poder utilizarlas en distintos tipos de dispositivos y valoran especialmente que estas aplicaciones dispusieran de los módulos asociados a avisos, casos prácticos, así como grabaciones de la asignatura. Resumiendo, la experiencia puede considerarse un éxito y un ejemplo de mobile learning multidispositivo. 


\section{BIBLIOGRAFÍA}

Al-Harrasi, H.; Al-Khanjari, Z. y Sarrab, M. [2015]: «Proposing a new design approach for m-learning applications», International Journal of Software Engineering and its Applications, 9 (11), págs. 11-24.

Amer, H. y Ibrahim, W. [2014]: «Using the iPad as a pedagogical tool to enhance the learning experience for novice programing students», Global Engineering Education Conference (EDUCON). 2014 IEEE, págs. 178-183.

Baturay, M. H. y Birtane, M. [2013]: «Responsive web design: a new type of design for web-based instructional content», Procedia-Social and Behavioral Sciences, 106 , págs. 2.275-2.279.

Bidin, S. y Ziden, A. A. [2013]: «Adoption and application of mobile learning in the education industry», ProcediaSocial and Behavioral Sciences, 90, págs. 720-729.

Brazuelo Grund, F. y Cacheiro González, M. L. [2010]: «Diseño de páginas web educativas para teléfonos móviles», EDUTEC. Revista Electrónica Tecnología Educativa, 32.

Ekanayake, S. Y. y Samarakoon, K. [2016]: «Support of mobile phones in a private network for science teaching», International Journal of Interactive Mobile Technologies, 10 (2), págs. 4-9.

Estudio Digital in 2016. Disponible en: http://wearesocial. net/.

Estudio IAB mobile 2015 (spain). Disponible en: www. iabspain.net/

Giemza, A.; Bollen, L.; Jansen, M. y Hoppe, H. U. [2012]: «An architecture for supporting heterogeneous multidevice learning environments», Wireless, Mobile and Ubiquitous Technology in Education (WMUTE). 2012 IEEE Seventh International Conference, págs. 67-71.

Gikas, J. y Grant, M. M. [2013]: «Mobile computing devices in higher education: student perspectives on learning with cellphones, smartphones \& social media», The Internet and Higher Education, 19, págs. 18-26.

Gordillo, A.; Barra, E.; Gallego, D. y Quemada, J. [2013]: «An online e-learning authoring tool to create interactive multi-device learning objects using e-infrastructure resources», Frontiers in Education Conference. 2013 IEEE, págs. 1.914-1.920.
Hargis, J.; Cavanaugh, C.; Kamali, T. y Soto, M. [2014]: «A federal higher education iPad mobile learning initiative: triangulation of data to determine early effectiveness»", Innovative Higher Education, 39 (1), págs. 45-57.

Isabwe, G. M. N.; Reichert, F. y Carlsen, M. [2013]: «Rethinking practices of assessment for learning: tablet technology supported assessment for learning mathematics», Teaching, Assessment and Learning for Engineering (TALE). 2013 IEEE International Conference on, págs. 155-159.

Kong, L. [2013]: «The development of mobile learning system based on the Android platform», Proceedings of the 2012 International Conference of Modern Computer Science and Applications, Springer Berlin HeideIberg, págs. 701-706.

Kousar, M. N. y Scholar, P. D. [2013]: Feasibility study of mobile learning in distance education, work paper of Distance, Non-Formal and Continuing Education Department, Islamabad: Allama Iqbal Open University.

Mamat, K. y Azmat, F. [2013]: «Mobile learning application for basic router and switch configuration on Android platform», Procedia-Social and Behavioral Sciences, 90 , págs. 235-244.

Martin, F. y Ertzberger, J. [2013]: «Here and now mobile learning: an experimental study on the use of mobile technology», Computers \& Education, vol. 68, October 2013, págs. 76-85.

Molnár, G. [2012]: «New learning spaces? M-learning's, in particular the iPad's potentials in education», Interactive Collaborative Learning (ICL). 2012 15th International Conference on, págs. 1-5.

Oberer, B. y Erkollar, A. [2013]: «Mobile learning in higher education: a marketing course design project in Austria», Procedia-Social and Behavioral Sciences, 93, págs. 2.125-2.129.

Ozuorcun, N. C. y Tabak, F. [2012]: «ls m-learning versus e-learning or are they supporting each other?», Procedia-Social and Behavioral Sciences, 46, págs. 299-305.

Peña, F. D. de la y Burgos, M. C. [2015]: «Modelo práctico de aplicación (app) para dispositivo móvil en las asignaturas universitarias de enseñanza a distancia. Experiencia con Android para la asignatura Dirección 
de Operaciones», EDUTEC. Revista Electrónica de Tecnología Educativa, 51.

Peña, F. D. de la y Prieto, S. [2014]: «Experiencia de aprendizaje mediante aplicación móvil en la enseñanza a distancia: un enfoque para la asignatura de grado Dirección de la Producción utilizando Android», VII Congreso Internacional Ikasnabar.

Peña, F. D. de la; García, C. B. y Simón, M. A. [2015]: «Mobile learning multidispositivo en la enseñanza universitaria mediante el uso de aplicaciones web. Experiencia en la asignatura Dirección de la Producción», EDUCADE. Revista de Educación en Contabilidad, Finanzas y Administración de Empresas, 6, págs. 59-79.
Sarrab, M.; Elgamel, L. y Aldabbas, H. [2012]: «Mobile learning (m-learning) and educational environments», International Journal of Distributed and Parallel Systems, 3 (4), págs. 31-38.

Setiabudi, D. H. y Tjahyana, L. J. [2013]: Mobile learning application based on hybrid mobile application technology running on Android smartphone and blackberry, doctoral dissertation, Jordania: Petra Christian University.

Yang, H. C. y Wang, W. Y. [2011]: «Facilitating academic service-learning with Android-based applications and ubiquitous computing environment», Ubi-Media Computing (U-Media). 2011 4th International Conference on, págs. 191-196.

\section{ANEXO}

\section{Encuesta realizada a los alumnos}

\section{CUESTIONARIO PARA ANALIZAR LA PERCEPCIÓN DEL ALUMNADO DE LAS WEB APPS}

1. Creo que la existencia de aplicaciones web (web apps) en las asignaturas mejoraría mi aprendizaje.
(1) Totalmente de acuerdo.
(4) En desacuerdo.
(2) De acuerdo.
(5) Totalmente en desacuerdo.
(3) Ni de acuerdo ni en desacuerdo.

\section{Estaría dispuesto a utilizar aplicaciones móviles web (web apps) para mis asignaturas.}
(1) Totalmente de acuerdo.
(4) En desacuerdo.
(2) De acuerdo.
(5) Totalmente en desacuerdo.
(3) Ni de acuerdo ni en desacuerdo.

3. La existencia de este tipo de aplicaciones móviles aportan un gran valor añadido a la asignatura.
(1) Totalmente de acuerdo.
(4) En desacuerdo.
(2) De acuerdo.
(5) Totalmente en desacuerdo.
(3) Ni de acuerdo ni en desacuerdo.

\section{4. ¿Para qué tipo de asignaturas crees que sería más útil la aplicación?}

Teóricas

Prácticas
Indiferente

Ninguna 


\section{CUESTIONARIO PARA ANALIZAR LA PERCEPCIÓN DEL ALUMNADO DE LAS WEB APPS}

.......

5. ¿Con qué dispositivos te gustaría acceder a la aplicación? Seleccionar todos los que se consideren:

Smartphone

Tablet

Smart TV

6. ¿Qué dispositivo piensas que es el más adecuado para utilizar este tipo de aplicaciones? Seleccionar únicamente uno:
Smartphone
Tablet
Smart watch
Smart TV

7. ¿Dónde piensas que utilizarías la aplicación? Selecciona todos los lugares que consideres:

$\begin{array}{ll}\square \text { Casa } & \square \text { Estando de viaje } \\ \square \text { Medios de transporte } & \square \text { En esperas (médico, transportes, etc.) } \\ \square \text { Trabajo } & \square \text { Otros } \\ \square \text { Lugares de ocio } & \end{array}$

8. Puntúa de 1 (menos) a 5 (más) la utilidad de cada una de las posibles características de la web appr:

\begin{tabular}{|c|c|c|c|c|c|}
\hline$\square 1$ & $\square 2$ & $\square 3$ & $\square 4$ & $\square 5$ & Calendario del aula \\
\hline$\square 1$ & $\square 2$ & $\square 3$ & $\square 4$ & $\square 5$ & Unidades de la asignatura \\
\hline$\square 1$ & $\square 2$ & $\square 3$ & $\square 4$ & $\square 5$ & Audios complementarios al manual \\
\hline$\square 1$ & $\square 2$ & $\square 3$ & $\square 4$ & $\square 5$ & Vídeos teóricos complementarios al manual \\
\hline$\square 1$ & $\square 2$ & $\square 3$ & $\square 4$ & $\square 5$ & Vídeos de casos prácticos \\
\hline$\square 1$ & $\square 2$ & $\square 3$ & $\square 4$ & $\square 5$ & Grabaciones de las sesiones virtuales \\
\hline$\square 1$ & $\square 2$ & $\square 3$ & $\square 4$ & $\square 5$ & Horario de tutorías \\
\hline$\square 1$ & $\square 2$ & $\square 3$ & $\square 4$ & $\square 5$ & Guía docente \\
\hline$\square 1$ & $\square 2$ & $\square 3$ & $\square 4$ & $\square 5$ & Test de autocomprobación \\
\hline$\square 1$ & $\square 2$ & $\square 3$ & $\square 4$ & $\square 5$ & E-mail directo al profesor \\
\hline$\square 1$ & $\square 2$ & $\square 3$ & $\square 4$ & $\square 5$ & Botón de llamada directa a la extensión del profesor \\
\hline$\square 1$ & $\square 2$ & $\square 3$ & $\square 4$ & $\square 5$ & Casos prácticos resueltos \\
\hline$\square 1$ & $\square 2$ & $\square 3$ & $\square 4$ & $\square 5$ & Casos prácticos autocorregibles \\
\hline$\square 1$ & $\square 2$ & $\square 3$ & $\square 4$ & $\square 5$ & Avisos de la asignatura \\
\hline$\square 1$ & $\square 2$ & $\square 3$ & $\square 4$ & $\square 5$ & Actividades interactivas \\
\hline$\square 1$ & $\square 2$ & $\square 3$ & $\square 4$ & $\square 5$ & Integrada con redes sociales (Twitter, Linkedin, etc.) \\
\hline$\square 1$ & $\square 2$ & $\square 3$ & $\square 4$ & $\square 5$ & Chat \\
\hline
\end{tabular}

9. ¿Qué ventajas crees que te daría la aplicación con respecto al aula de la asignatura?

10. ¿Qué otros aspectos incluirías en este tipo de aplicaciones? 


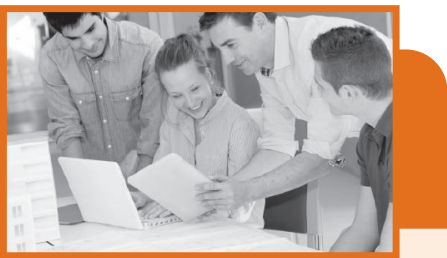

\section{CM \\ curso \\ monográfico}

\section{Gestión de Redes Sociales}

en la Empresa (Community manager)

DURACIÓN: Clases on line [4 créd.]: 4 meses (inicio: octubre y febrero).

\section{CM \\ curso \\ monográfico}

ON LINE OL.

\section{Habilidades y}

\section{Competencias Directivas}

DURACIÓN: Clases on line [5 créd.]: 3 meses (inicio: octubre, febrero y mayo).

\section{CM \\ curso \\ monográfico}

ON LINE OL

\section{Diseño de Programas Formativos e-Learning \& b-Learning}

DURACIÓN: Clases on line [3 créd.]: 3 meses (inicio: octubre, febrero y mayo).

\section{CM \\ curso \\ monográfico}

ON LINE OL

\section{Formador de Formadores on Line}

DURACIÓN: Clases on line [ 3 créd.]: 3 meses (inicio: octubre, febrero y mayo).

\section{CM \\ curso
monográfico}

ON LINE OL

\section{Fundamentos de Dirección de Recursos Humanos}

DURACIÓN: Clases on line [3 créd.]: 3 meses (inicio: octubre, febrero y mayo).

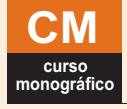

ON LINE OL

\section{Dirección Estratégica y Cuadro de Mando de Recursos Humanos}

DURACIÓN: Clases on line [3 créd.]: 3 meses (inicio: octubre, febrero y mayo).

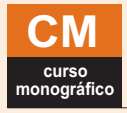

ON LINE OL

\section{Recursos Humanos 2.0 y Organizaciones 2.0}

DURACIÓN: Clases on line [3 créd.]: 3 meses (inicio: octubre, febrero y mayo).

\section{CM \\ curso \\ monográfico}

ON LINE OL

\section{Gestión de la Formación}

DURACIÓN: Clases on line [4 créd.]: 3 meses (inicio: octubre, febrero y mayo). 